vol. 26 - $n^{\circ} 2 \mid 2010$

Numéro ouvert

\title{
Les Grecs du Cameroun, une diaspora d'entrepreneurs (1950-2007)
}

Greeks of Cameroun, an Entrepreneurial Diaspora (19050-2007)

Los Griegos del Camerún, una diáspora de empresarios (1950-2007)

\section{Nicolas A. Métaxidès}

\section{(2) OpenEdition}

Journals

\section{Édition électronique}

URL : https://journals.openedition.org/remi/5152

DOI : 10.4000/remi.5152

ISSN : $1777-5418$

\section{Éditeur}

Université de Poitiers

\section{Édition imprimée}

Date de publication : 1 septembre 2010

Pagination : 169-189

ISBN : 978-2-911627-55-2

ISSN : 0765-0752

\section{Référence électronique}

Nicolas A. Métaxidès, «Les Grecs du Cameroun, une diaspora d'entrepreneurs (1950-2007) », Revue européenne des migrations internationales [En ligne], vol. 26 - n² | 2010, mis en ligne le 01 septembre 2013, consulté le 14 avril 2022. URL : http://journals.openedition.org/remi/5152 ; DOI : https://doi.org/ 10.4000/remi.5152 


\title{
Note de recherche
}

\section{Les Grecs du Cameroun, une diaspora d'entrepreneurs (1950-2007)}

\author{
Nicolas A. MÉTAXIDÈS ${ }^{*}$
}

T 'Afrique méditerranéenne a, depuis très longtemps, attiré les Grecs car ¿elle faisait partie de l'Empire ottoman et représentait un pôle important de la diaspora marchande hellénique (Soulogiannis, 1999 et 2001 ; Kazdaghli, 2000 ; Agathagelidou, 2003). L'Afrique au sud du Sahara par contre ne comptait qu'un nombre relativement faible de migrants grecs. La majorité d'entre eux travaillait dans les mines, les chemins de fer et l'industrie en Afrique du Sud. Avec le développement du secteur secondaire et tertiaire dans cette région des migrants vont aussi occuper des emplois libres (Mantzaris, 1981, 1984, 1987). Ce n'est que dans les années d'après-guerre (1950) et dans les années suivant les indépendances (années 1960) que la migration des Grecs en Afrique subsaharienne va prendre un caractère relativement massif ${ }^{1}$. Notre objectif dans cet article est d'interroger la présence hellénique en Afrique Centrale et particulièrement au Cameroun. Il s'agira d'étudier les Grecs du Cameroun au prisme de leurs itinéraires, de leur organisation sociale (église, communauté, langue), de leurs activités économiques (professions, activités entrepreneuriales). Nous nous sommes particulièrement demandé comment les institutions liées à la culture des migrants grecs d'une part, et l'environnement du pays qui les a reçus d'autre part ont influencé leurs choix personnels ou collectifs. Quelles sont les raisons de l'insertion de ces Grecs du Cameroun dans le réseau diasporique en Afrique subsaharienne, et quelles sont les conditions qui ont éloigné certains d'entre eux, les poussant à rentrer au pays d'origine?

\footnotetext{
* Chercheur à l'université de Péloponnèse (département de Sciences Politiques et Relations Internationales), 33 rue Péloponnissou, 16345, Athènes, Grèce ; nickirene.met@gmail.com

1 Le nombre d'études sur la diaspora hellénique en Afrique subsaharienne (excepté l'Afrique du Sud) est peu important. Les travaux de Trevaskis (1960), Matsoulas (1975) et Petronoti (2000) traitent de la présence hellénique dans les pays de la Corne d'Afrique ; ceux de Lazidou (2004) de la présence hellénique en Afrique orientale ; ceux de Métaxidès (2003, 2004) concernent le Cameroun et ceux de Markakis (1998) et Kazakos et al. (1998) sont des synthèses concernant la présence hellénique en Afrique subsaharienne.
} 


\section{ITINÉRAIRES MIGRATOIRES}

Nous débuterons ce rétrospectif historique sur la présence grecque d'aprèsguerre au Cameroun, en nous appuyant sur des extraits d'entretiens de Grecs établis au Cameroun ${ }^{2}$.

« Je suis arrivé au Cameroun en 1952, à l'âge de dix-sept ans. Le frère de ma mère m'a amené, il vivait au Cameroun depuis 1925 et lui aussi avait été amené par son oncle. Mon oncle avait des plantations de bananes et de café à Loum ${ }^{3}$ et il possédait aussi plusieurs magasins - commerce de denrées alimentaires, épiceries

- à Douala : j'ai travaillé à Douala dans l'un de ses magasins. En 1953, je suis

devenu responsable de notre filiale à Bafia. À cette époque il n'y avait ni eau, ni électricité. Je faisais le commerce du cacao et en même temps je gardais le magasin.

C'était doublement difficile car je ne parlais pas français. Je suis resté en tant qu'employé jusqu'en 1957. Avec mes économies j'ai acheté le magasin et je l'ai géré

personnellement. Le magasin vendait de tout (vêtements, chaussures, fournitures scolaires, etc.) J'achetais les produits à des représentants à Douala et à Yaoundé. À cette époque les déplacements étaient une véritable aventure, en particulier lorsqu'il pleuvait. Avec des conditions normales nous faisions l'aller à Bafia depuis Yaoundé

en douze heures. Nous traversions les fleuves Sanaga et Mbam avec deux bacs.

Depuis 1980 un pont a été construit au confluent des deux rivières. J'ai acheté en 1957 avec les économies faites à Bafia, un terrain à Yaoundé de 1960 mètres carrés, où j'ai établi un magasin en 1959. J'ai loué ce magasin jusqu'en 1970. En 1967 je suis descendu de Bafia à Yaoundé, afin de remplacer un employé grec de mon oncle qui partait en vacances. Après son retour, je ne suis pas revenu à Bafia. J'ai ouvert trois magasins à Yaoundé et j'y ai travaillé jusqu'en 1971. Je me suis occupé ensuite

de construire mon propre logement sur le terrain que j'avais acheté. Dès lors, $j$ 'ai commencé une nouvelle carrière. J'ai investi dans les matériaux de construction qui

étaient très demandés à cette époque étant donné que dans ce secteur (à la sortie de la ville) le négoce des vêtements n'était plus rentable. Je vendais des pelles, des pioches, du fer, du ciment, des casseroles, etc. aux paysans. À cette époque j'ai gagné beaucoup d'argent. J'ai fait ce travail jusqu'en 1984, année durant laquelle ce terrain fut exproprié. Je suis encore dans les procédures judiciaires pour être indemnisé. Depuis, ce sont les loyers qui me font vivre » (G.S.).

«Je suis venu fin 1966. J'avais deux oncles au Cameroun, un à Yaoundé (celui qui est venu en premier) qui était tailleur tandis que le second s'activait dans le commerce du cacao et le commerce général ; il possédait aussi une boulangerie (un four à bois à cette époque) dans la ville de Saa. Il achetait le cacao auprès des producteurs à qui il avançait souvent l'argent-avant la saison de la récolte - pour qu'ils puissent acheter des produits de base, des médicaments, etc. À une époque,

l'État a mis en place des coopératives où les cultivateurs de café et de cacao vendaient leur production. Dans sa boulangerie, il utilisait de la levure fraîche qu'il faisait venir de Yaoundé où il l'achetait chez Despotakis qui avait une épicerie

là-bas. Nous avons essayé aussi de travailler avec son fils, mais il m'a escroqué et j'ai été forcé de continuer à travailler seul. De 1978 à 1984, je faisais du commerce

2 Les interviews ont eu lieu en Grèce et au Cameroun. La recherche de terrain au Cameroun s'est déroulée en janvier 2003 et entre septembre et novembre 2007 à Douala, Nkongsamba, Obala, Saa, Bafia, Mbalmayo, Ebolowa et Sangmélima.

3 Sur la route reliant Douala à Nkongsamba. 
(denrées alimentaires, bières, vins) et je produisais du cacao (à Saa où je travaillais avec la coopérative). Nous faisions les intermédiaires entre la coopérative et l'exportateur. En 1979 j'ai monté une autre boulangerie à Saa, en coopération avec un Grec. J'ai arrêté le commerce de cacao en 1984, époque où je suis resté en même temps seul à la boulangerie puisque la collaboration avec mon associé était devenue problématique. Après 1984, je suis resté de façon définitive à Yaoundé ; les enfants et mon épouse étaient en Grèce. Je me suis aussi occupé du transport. J'ai fait du transport de marchandises (du cacao, du café de Yaoundé à Douala, du ciment et d'autres produits de Douala à Yaoundé). J'avais quatre camions. Je chargeais du

bois de placage depuis Mbalmayo pour Douala et au retour je transportais des marchandises. J'ai travaillé jusqu'en 1997 avec Stamatiades, Kritikos, Jakovides,

Christodoulides (CACEP-cacao). J'ai acheté trois autres camions. Mais j'ai été malchanceux, $j$ 'ai eu des problèmes (accidents), j'en ai perdu deux et j'ai vendu le troisième. En plus de la boulangerie, je faisais aussi de l'exportation de bois. Même si la région n'est pas réputée pour sa richesse forestière, il y a du bois. Je l'achetais aux alentours et je le coupais en planches (raboté) aux dimensions que voulait l'exportateur qui était français. Ce travail n'était pas légal, je l'ai exercé sans autorisation jusqu'en 2000. Aujourd'hui, je conserve seulement la boulangerie » (T.G.). «En Grèce, je travaillais comme marchand de poisson. Nous gagnions de l'argent à cette époque, mais nous étions jeunes, et nous ne savions pas le garder. Je suis donc venu au Cameroun en 1971. C'est mon oncle qui m'a amené, lui qui était venu au Cameroun avant 1960 (aux environs de 1958). Il produisait du cacao. Moi aussi j'ai fait des affaires avec le cacao. J'ai appris avec mon oncle. Je ne suis resté avec lui que très peu de temps (huit mois), j'ai appris la langue et le travail. Mon oncle était à Ngomedzap (au sud de Mbalmayo) et moi à Lolodorf (à l'est de Kribi et au nordouest d'Ebolowa). Lorsque j'ai commencé à travailler le cacao, ce dernier appartenait à l'État. Le service de l'État fixait un prix unique pour tout le Cameroun, jusqu'à il y a six ou sept ans. Trois qualités de cacao existaient. Grade 1:500 CFA; grade 2: $450 \mathrm{CFA}$; hors standard: $100 \mathrm{CFA}$; résidu (inutilisable). À cette époque-

là, il avait des marchés organisés et les dates de leurs ouvertures étaient fixées par les préfectures. Il existait des contrôleurs d'État qui contrôlaient les qualités de cacao sur le marché. Nous allions avec nos acheteurs et nos camions faire nos achats auprès des cultivateurs. Nous amenions le cacao sur le marché là où il y avait des dépôts et c'est là que se faisaient les transactions. Le contrôle de chaque cultivateur se faisait selon la procédure suivante : le contrôleur "touchait un petit cadeau" afin que dans son estimation la qualité grade 1 passe en grade 2 et fasse ainsi baisser le prix. Le cultivateur était impuissant puisque le contrôleur était un représentant de l'État et par conséquent c'était lui qui commandait, ses décisions ne pouvaient donc être remises en cause. Ainsi, le "hors standard" était représenté dans une proportion très importante qui atteignait aisément les trente sacs sur 150, soit $20 \%$. Cette procédure a continué plus au moins jusqu'en 1985-1990. Après 1990 le prix du cacao a été libéré. Aujourd'hui il est défini par la bourse internationale et c'est la raison pour laquelle il varie énormément. Il y a longtemps, les exportateurs étaient principalement grecs, comme Kritikos, Mavromatis, Christodoulidis $(C A C E P)$, mais il y avait aussi une société d'intérêts juifs (SOCAPA). Dernièrement il y avait aussi CITAGRI, une société créée par un neveu de Kritikos. Aujourd'hui, les exportateurs sont français, belges, camerounais. Après la libération des prix, la concurrence sur le marché du cacao est devenue très forte et beaucoup d'acteurs ont abandonné ce marché. Je travaillais en tant que directeur à la CACEP. J'y suis resté de 1982 à 1995. Jusqu'en 1982 je travaillais seul, mais aussi avec Kritikos à Ebolowa où j'ai aussi créé une boulangerie. À cette époque la boulangerie consti- 
tuait une autre activité rentable. En 1980 j'ai vendu la boulangerie à un Bamiléké. Lorsque la CACEP a fait faillite, j'ai continué seul en achetant les bâtiments qu'elle possédait à Sangmélima. L'achat du cacao se fait sur un rayon de 150 kilomètres.

À Sangmélima j'ai le commerce du cacao, une boulangerie et une quincaillerie. Pour l'achat du cacao, nous avons environ trente voitures. Mes fils travaillent à la boulangerie. Ils ne restent pas ici de façon permanente, mais ils viennent à tour de rôle » (S.K.).

\section{LA PRÉSENCE GRECQUE AU CAMEROUN}

Les Grecs du Cameroun constituent une part importante de la présence grecque dans l'Afrique du centre-ouest. À partir de la seconde moitié du XIXe siècle, on peut constater des installations isolées de Grecs en Afrique occidentale. La plus caractéristique est celle de Georges Zochonis, originaire d'un village près de Sparte, qui arrive vers 1870 en Afrique occidentale et plus particulièrement en Sierra Leone, avec comme motif s'enrichir. En 1879, il fait la connaissance de Georges Paterson originaire d'Écosse. Ils travaillent tous deux à Freetown dans une maison commerciale britannique, la Trading Company of Fisher \& Randall ; et ils se lient d'amitié. En 1879 ils décident de fonder une société - la Paterson, Zochonis \& Co. Limited - qui sera connue dans la région : "The two Georges ». Leurs activités économiques couvrent toute l'Afrique du centre-ouest. $\mathrm{Au}$ Cameroun, la Paterson-Zochonis (PZ) s'installe en 1922 à Douala (Mainet, 1985 : 228). La présence de la PZ renforce considérablement le flux migratoire hellénique vers le Cameroun, surtout après la Deuxième Guerre mondiale. La PZ utilise presque exclusivement des employés grecs, qui viennent principalement de la région d'origine de Zochonis, la Laconie. Par la suite, d'autres Grecs précurseurs seront à l'origine de l'immigration de leurs compatriotes (frères, parents mais aussi concitoyens originaires du même village).

Au Cameroun, l'arrivée des Grecs s'est faite essentiellement en deux étapes. La première correspond à la période de l'entre-deux-guerres (décennie 1920) tandis que la seconde date de la fin de la Deuxième Guerre mondiale (décennie 1950). Les Grecs qui sont arrivés lors de la première phase étaient principalement originaires du Pont-Euxin et de l'Asie Mineure. Après « le Désastre d'Asie Mineure » et l'Échange des populations (1923), plusieurs d'entre eux sont allés s'installer à Marseille ${ }^{4}$. À cette époque les transports maritimes prédominaient encore et les villes de Bordeaux et Marseille constituaient les principaux ports carrefours de la région. La France a été l'une des principales destinations (parfois aussi pays de transit) pour les immigrés grecs ${ }^{5}$. La seconde période se caractérise principalement par l'immigration provenant de Macédoine orientale et de

4 La communauté hellénique de Marseille existe depuis le XIXe siècle (Paris, 2001 : 23-42).

5 « La France a accueilli des Grecs de Smyrne ou d'Anatolie... Ceux qui provenaient des grandes villes de l'Orient étaient instruits et souvent assez riches. En s'installant dans des villes d'Occident, ils se sont intégrés rapidement dans la vie économique et intellectuelle des sociétés d'accueil. D'autres ont préféré un comportement plus typiquement diasporique. Ils se sont installés dans des pays d'Amérique latine ou d'Afrique où leurs chances de s'enrichir et de faire partie de l'élite locale étaient plus fortes » (Prévélakis, 1996 : 61). 
Thrace, ainsi que du Dodécanèse ${ }^{6}$.

À partir des années 1950, la communauté grecque du Cameroun a sensiblement augmenté : d'environ $20 \%$ en moyenne par an jusqu'au début des années 1970, au point d'atteindre 2200 personnes, nombre maximal de la présence grecque dans le pays. Nous estimons que dans la bibliographie grecque de l'époque leur nombre a été surévalué en le fixant à 3000 au début des années 1960 (Markantonatos, 1965). Cette surestimation est due au fait que le recensement prend en compte toutes les formes de migrations grecques (et gréco-chypriotes) vers le Cameroun sans exclure les migrations saisonnières d'individus qui retournaient dans leur pays d'origine après les périodes de récolte (cacao et café) ${ }^{7}$. À la fin de 1951, ils représentent $3 \%$ des étrangers non africains (Lembezat, 1954 : 71) et un peu moins de $5 \%$ en 1976 (Mainet, $1985: 87$ ).

L'immigration des Grecs d'Égypte, forcés de quitter ce pays après l'arrivée au pouvoir de Nasser et de sa politique de nationalisation, a joué un rôle décisif dans l'élargissement de la communauté grecque du Cameroun. Le témoignage que nous a livré Konstantinos Antoniadis - consul honoraire de Chypre au Cameroun - constitue une éloquente illustration de l'émigration des Grecs d'Égypte vers le Cameroun :

«Mon père était d'origine chypriote, né au Caire. Mon grand-père était un milliardaire au Caire. Il avait deux usines de production de beurre qui fabriquaient presque le quart de la production et de la consommation en Égypte. Mon père avait fait des études d'ingénieur chimiste et était spécialisé dans les matières grasses, parce que son objectif était de continuer, après ses études, à travailler dans les usines de son père. Le frère de mon père, Christo, a fait la connaissance à Marseille d'une fille de la famille Koulaxidis. Les deux jeunes, ayant pressenti les événements qui allaient arriver en Égypte, partent en 1951 au Cameroun avant les nationalisations. Leur décision a fortement contrarié la famille en Égypte. Mon père a pris connaissance de leur décision à Marseille lors de son départ pour le Canada où vivait aussi une partie de la famille. Christo a finalement convaincu son frère (mon père) de le suivre au Cameroun au lieu du Canada. Il est arrivé finalement en Afrique sous l'incitation de son frère, mais aussi après l'invitation de Talidis, un oncle du côté de leur mère qui était lui aussi un homme d'affaires qui avait bien

6 Nous avons interviewé quatre-vingt-dix-huit individus, dont cinquante-cinq au Cameroun (vingtdeux vivent à Douala, dix-neuf à Yaoundé, deux à Nkongsamba, trois à Sangmélima, un à Mbalmayo, quatre à Ebolowa, quatre à Obala), quarante-trois en France et en Grèce (quatre à Salonique, dix à Rhodes, vingt-sept à Athènes et deux à Chypre). Les Grecs du Cameroun, qui ont immigré définitivement en Grèce, ont fondé en 1995 l'Association des Grecs du Cameroun de retour en Grèce. En 2004 ils ont changé le titre de leur association : l'Association des Grecs du Cameroun. L'ex-président de l'Association nous dit que ce changement d'intitulé correspond au désir de mettre l'accent sur un passé lié au Cameroun que les Grecs de retour considèrent comme leur deuxième patrie. L'association compte environ 180 membres. Nous estimons le nombre total des Grecs du Cameroun - au Cameroun et ailleurs - entre 500 et 600 personnes ; 150 à 200 résident au Cameroun, 180 à Athènes, entre soixante-dix et quatre-vingts à Rhodes, et entre soixante et quatre-vingts au Nord et au Nord-est de la Grèce (Salonique, Drama, Kavala). D'après nos enquêtes 29,6\% des enquêtés sont originaires du Nord et du Nord-est de la Grèce, 23,5\% du Dodécanèse, $12,2 \%$ du Péloponnèse.

7 La saison du cacao commence en septembre et se termine début janvier, tandis que celle du café se poursuit jusqu'en mars. 
réussi. La société de Talidis sous l'appellation C.C.C. (Compagnie Commerciale

Chypriote) $)^{8}$ était mise en place en collaboration avec Katsaris et d'autres. Mon père, en tant qu'expert, a créé une savonnerie à Bassa (zone industrielle de Douala).

Lorsque nous sommes arrivés le 14 janvier 1951 (j'avais alors quatre ans) rien $n$ 'existait encore dans la région. Nous vivions dans une baraque faite entièrement en herbe et en paille. L'unique élément de type occidental de cette habitation était le sol en ciment. Tout le reste était fabriqué en bambou, en poutre et en paille, les cloisons étaient toutes en bambou et les fenêtres n'avaient pas de volets. Je me rappelle que ma mère craignait beaucoup les lézards et les scorpions. Le premier chantier important que mon père a réalisé était le barrage d'eau, qui existe toujours aujourd'hui sur le site de l'usine. Il est vrai que la fabrication du savon exige beaucoup d'eau et en particulier de l'eau sous pression. Ses connaissances en matière de techniques de retenue d'eau, il les avait acquises à Chypre, aux côtés des Anglais, à l'époque où Chypre se trouvait sous la tutelle britannique, puisque sa mission était la réutilisation, dans le cadre des projets industriels, du matériel de guerre abandonné par les Allemands sur les champs de combats et tombé aux mains des Anglais. Au Cameroun, il a été le premier à fabriquer du savon en barre.

Le savon était versé dans des boîtes au sol et ensuite il était coupé à la main avec de grands ciseaux. Beaucoup de soucis ont suivi, le décès du père Katsaris et de Talidis. Les choses ne sont pas bien passées entre les associés et mon père est parti. Il a donné sa part et il est parti. La C.C.C. existe encore aujourd'hui mais elle a été achetée par un Camerounais qui se nomme Fadil, imposé par le président Ahmadou Ahidjo"; qui est intervenu personnellement auprès de Katsaris pour lui céder $5 \%$ des actions. Progressivement, Fadil a acheté l'ensemble de la société, étant donné que mon père est parti. Lorsqu'il a acquis la majorité des actions, Fadil a changé l'appellation de la société. Mais comme la société était toujours connue sur le marché sous le nom C.C.C., ils ont été obligés de maintenir le sigle et ils ont trouvé comme solution de la nommer Complexe Chimique Camerounais, au lieu de Compagnie Commerciale Chypriote, qui était une marque déposée grecque. Fadil a conservé à son poste le directeur général, Monsieur Papadopoulos ${ }^{10} »$.

L'émigration des Grecs vers l'Afrique subsaharienne ainsi que vers le Cameroun a pris une plus grande ampleur surtout après 1955. Plusieurs raisons ont contribué à ce phénomène, par exemple l'élargissement constant du socle migratoire, à savoir le nombre des pionniers qui vont attirer à leur tour plusieurs membres de leurs familles, compatriotes et amis. En effet, il est important de signaler que les immigrés d'après-guerre, dans la plupart des cas, amènent aussi avec eux au Cameroun leurs familles, tandis que les immigrés d'avant-guerre étaient dans leur grande majorité célibataires. Bon nombre parmi eux rentrent en Grèce à la recherche d'une épouse originaire du pays d'origine. Un autre facteur qui a contribué à amplifier cette émigration est l'amélioration des conditions de voyage grâce au développement du transport aérien qui remplace progressivement le

\section{Fondée en 1944.}

9 Ahidjo, Ahmadou (1924-1989) : premier président du Cameroun (1960-1982). Il a démissionné en 1982 en laissant son poste à Paul Biya.

10 Consul général du Cameroun en Grèce depuis 1986. 
voyage en bateau ${ }^{11}$.

Durant les années 1970, la position de la communauté grecque au Cameroun se renforçait suite à la crise pétrolière et à la politique nationaliste du président Ahidjo, conduisant les grandes maisons françaises à abandonner progressivement le pays. Les Grecs qui, jusque-là se fournissaient exclusivement auprès des maisons françaises et vendaient ensuite au détail, commencent à se rendre eux-mêmes en Europe pour acheter les marchandises avec lesquelles ils allaient alimenter les marchés camerounais.

Jusqu'à la fin de la décennie 1970, le Cameroun constituait un modèle de développement parmi les pays d'Afrique. La découverte des gisements pétrolifères dans le golfe de Guinée, à la fin de l'époque Ahidjo, donnait un nouveau souffle au pays à tel point qu'on parlait de «miracle camerounais » et que l'on considérait ce pays comme une véritable oasis de prospérité, située au milieu d'un continent frappé par la misère. La communauté économique internationale comptait le Cameroun parmi les vingt pays les plus sûrs pour les investissements étrangers. Au milieu des années 1980, le Cameroun est entré dans une crise prolongée (Ngandjeu, 1988 ; Aerts et al., 2000). Au Cameroun, la conjoncture économique favorable (jusqu'au milieu des années 1980), a contribué à l'augmentation de la population grecque. Cependant, la crise économique qui a suivi a eu des répercussions sur cette communauté, dans un premier temps après 1985 puis, après 1994 à cause de la dévaluation du franc CFA, période durant laquelle le nombre de Grecs est tombé à 450 individus vivant principalement à Yaoundé (160), à Douala (240) et à Nkongsamba (10) (Kazakos, 1998 : 338). Aujourd'hui leur nombre ne dépasse pas les 250 individus en incluant aussi ceux qui vivent dans d'autres pays, par exemple en France ou en Grèce, et qui viennent au Cameroun périodiquement afin de superviser leurs affaires (Métaxidès, 2004 : 327-341).

\section{LES GRECS ET LE SYSTÈME ÉCONOMIQUE COLONIAL}

L'activité grecque en Afrique est étroitement liée aux plantations (café, agave, cacao, bananes, coton, etc.), à l'exploitation du bois et des richesses minières, au commerce général (importations d'équipements électriques, de tissus, d'articles de quincaillerie, etc.). Les grossistes grecs utilisaient des intermédiaires qui négociaient et achetaient les produits agricoles. Ces intermédiaires étaient des Grecs qui venaient en Afrique en tant qu'immi-

11 «Lorsque je suis arrivée au Cameroun en 1952, j’ai voyagé d'abord avec le bateau "[Aiolia]" du Pirée jusqu'à Marseille. À Marseille nous avons fait des vaccins supplémentaires et avec un avion à quatre moteurs, nous sommes parvenus au Cameroun (Douala) après douze heures de voyage. Nous avons fait un arrêt à Alger pour le ravitaillement et pour le dîner et à Niamey au Niger également pour le ravitaillement et pour le petit-déjeuner, avant de prendre la route pour notre destination finale qui était Douala au Cameroun » (entretien réalisé en 2007 avec Madame Comissa Grigoriadou). "Avec une valise en carton remplie de peu de choses, nous sommes partis du Pirée le 12 mars avec pour destination... Alexandrie... le bateau s'appelait "Cairo City". Notre deuxième étape était Le Caire. L'avion était un Dakota de British Airways... chaque deux heures presque l'avion s'arrêtait pour se ravitailler. Notre premier arrêt a eu lieu à Khartoum (Soudan)... à Maiduguri (Nigeria), à Lagos (Nigeria) ... nous sommes arrivés à Douala le12/4/1946... » (Vlahos, 1999 : 18-19). 


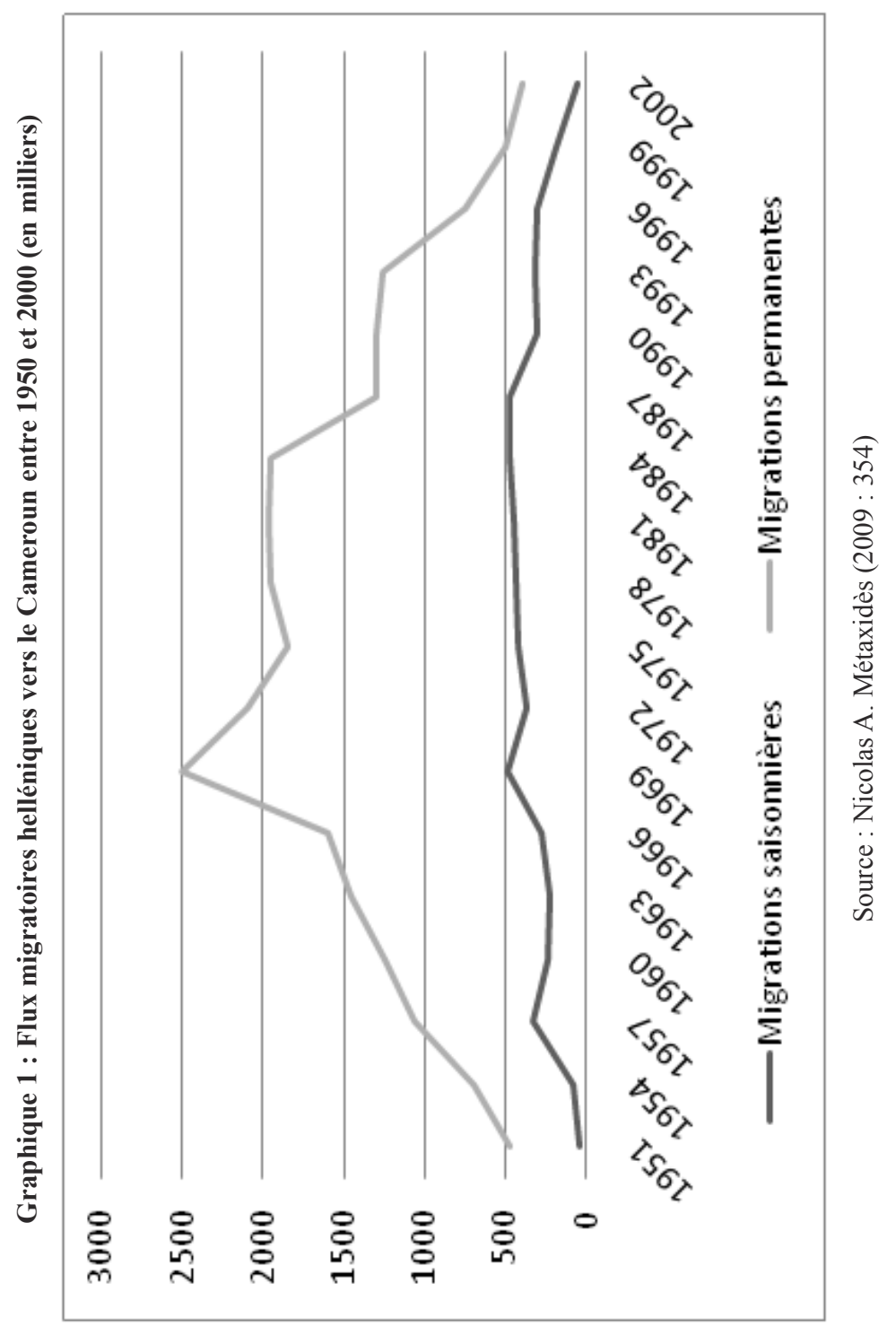


grés saisonniers pendant la période des récoltes. L'exploitation des richesses forestières et minières, dans le cas du Cameroun, se faisait jusqu'en 1914 par les Allemands qui exploitaient aussi les plantations qu'ils avaient créées à l'ouest du pays, notamment dans la région du mont Cameroun où les terrains volcaniques étaient très favorables aux cultures. Plus tard, après la défaite allemande durant la Première Guerre mondiale, l'exploitation des richesses du pays est tombée aux mains des Anglais et des Français ${ }^{12}$. Les Grecs ont voulu garder une attitude relativement amicale face aux autochtones à qui ils vendaient par ailleurs leurs marchandises (produits industriels tels que : articles électriques, tissus, etc.), en les considérant cependant comme inférieurs ( sans intérêt et motivation pour le progrès »). Sur les publicités de l'époque, on peut voir la distinction qui était faite entre les produits vendus aux blancs et ceux destinés aux indigènes (Sakéllaridès, 1960). Naturellement les avis des autochtones sur le rôle joué par les Grecs au Cameroun divergent. Certains considèrent que le Grec n'ayant jamais été un colonisateur était par conséquent plus proche des noirs, d'autres au contraire le placent au même niveau que les Français en le considérant comme le fer de lance de l'exploitation coloniale dans les secteurs où les colons eux-mêmes n'étaient pas compétitifs. Célestin Akoa-Ngbwa nous dit :

«Les Grecs sont pacifiques et ils respectent les lois du pays qui les accueille. Personne jamais n'a cherché à me flouer au niveau de mon salaire. Le salaire était fixé par la direction et on ajoutait les primes au rendement. J'ai travaillé vingt-six

années avec eux. [...] Christodoulidès avait sous sa protection de nombreuses familles camerounaises. Il était généreux et il leur faisait des cadeaux. [...] Les

Grecs ont beaucoup aidé le Cameroun. De nombreux enfants d'employés qui travaillaient pour les Grecs sont allés à l'école et certains parmi eux sont même devenus ministres. Les Grecs, et dans une moindre mesure les Libanais, ont construit des bâtiments, ont investi. Au contraire, les Français n'ont rien fait. Je ne dis pas cela parce que j'ai travaillé chez les Grecs mais parce que c'est la vérité. Les Grecs ne faisaient pas de politique et respectaient leurs promesses ».

On peut lire cependant un autre témoignage bien différent ${ }^{13}$ :

«De nombreuses familles grecques (Despotakis, Mavromatis, Dimitropoulos, Kalafatas) sont venues en Afrique à l'époque du colonialisme ${ }^{14}$ et ont profité de la générosité des Français qui leur ont confié l'exploitation du cacao. Aujourd'hui, les richesses accumulées de ces charlatans ont souvent été construites sur la perfidie et la malveillance de ces premières familles qui sont arrivées sans avoir le nécessaire, contrastant avec l'opulence des colons français. En ayant le monopole du marché du cacao, ils pouvaient, en fonction de leurs intérêts, acheter la production de ce dernier, ou encore l'estimer de mauvaise qualité et l'envoyer tout simplement au feu, en faisant ainsi varier les prix. Ils ne s'intéressaient pas à la qualité du produit, la seule chose qui comptait était leur intérêt. Souvent ils risquaient leur vie puisque les réactions des noirs face à l'injustice étaient dans certains cas très fortes. Le litige entre le commerçant grec et le vendeur local de cacao se réglait avec l'intervention

12 La France a pris le contrôle du Cameroun oriental, tandis que le Cameroun occidental se trouvait sous l'autorité de la Grande-Bretagne.

$13 \mathrm{http} / /$ www.africultures.com/index.asp?menu=forum\&no=1491\&no_theme=11.

14 Ces familles sont arrivées au Cameroun dans les années 1930. 
de l'administration coloniale. Cette dernière se chargeait de trouver le coupable.

Les croyances religieuses que les missionnaires occidentaux ont transmises durant

la période coloniale permettaient facilement de découvrir le coupable. Il suffisait que celui-ci aille se confesser au prêtre pour finir immédiatement entre les mains des

bourreaux».

Mongo Béti dans le roman La ville cruelle, met dans la bouche de son héros les mots suivants :

« Demain, proposa-t-il, je m'en vais à la ville pour vendre mon cacao aux Grecs. J'espère que ces fils de voleurs me donneront suffisamment d'argent pour mes affaires » (Mongo Béti, 1971 : 14).

Et plus loin, «Le centre commercial, comme on l'appelait; on aurait tout aussi bien fait de l'appeler le centre grec. Tout le long des rues, les enseignes sonnaient grec : Caramvalis, Despotakis, Pallogakis, Mavromatis, Michalidès, Staberidès,

Nikitopoulos, etc. Leurs boutiques étaient construites à rez-de-chaussée avec des vérandas [...] elles vendaient tout [...] et c'est chez eux que vous trouveriez les prix les plus bas. Et c'est dans leur maison que vous trouveriez la meilleure marchandise. On voyait rarement le patron grec, sauf pendant la saison $d u$ cacao $^{15}$. [...] Les paysans accouraient avec leurs charges, s'amassaient devant le Levantin. Et plus il $y$ en avait et plus il en venait, et plus il était facile pour Pallogakis de faire baisser

progressivement et insensiblement les taux et de commettre d'autres fraudes »

(Mongo Béti, 1971 : 18-19).

Les motivations de la présence grecque en Afrique étaient principalement économiques. Les colons contrôlaient la capitale et en tant qu'administrateurs de l'économie, ils contrôlaient les postes qui nécessitaient des connaissances techniques, en laissant les travaux non qualifiés et manuels aux autochtones (Kaptue, 1986 : 9). Les pionniers se sont lancés dans le commerce des produits agricoles à grand rendement (achat et stockage de café, de cacao, de bananes). Ils vivaient dans des régions isolées ${ }^{16}$ près des cultures de cacao et de café et parallèlement ils ont développé une activité commerciale dans les villes (principalement au port de Douala) en exportant des produits agricoles et en introduisant toute sorte de produits transformés (tissus, articles électriques, meubles, etc.).

\section{QUI SONT LES IMMIGRÉS ?}

Le portrait type de l'immigré grec en Afrique « est un jeune agriculteur, sans culture ni qualification » (Markakis, 1998 : 29). Quand il arrive, il a entre vingt et trente ans, il est célibataire et il a très peu d'éducation et de qualification professionnelle. Cette description, bien qu'elle ne soit pas loin de la réalité, n'est pas totalement conforme à l'image des immigrés grecs du Cameroun, en particulier de ceux qui sont arrivés entre les

15 Du début août jusqu'à mi-février, période pendant laquelle se fait la collecte, le séchage ainsi que la vente du cacao.

16 Les Grecs menaient un train de vie qui les rapprochait de celui des autochtones. Ils apprenaient la langue de la région et se familiarisaient avec la culture des populations locales. Seuls les Bamiléké ont pu être des adversaires particulièrement dans le secteur commercial. 
années 1920 et la veille de la Seconde Guerre mondiale, en majorité des Grecs originaires du Pont-Euxin et de l'Asie Mineure ${ }^{17}$.

Le Cameroun est un pays difficile qui exige - comme d'ailleurs n'importe quel autre pays d'Afrique noire - beaucoup de courage et de sacrifices de la part de l'immigrant. L'immigré grec qui vient au Cameroun suit généralement les traces d'un proche qui peut être un parent, un ami ou encore quelqu'un du même village. Il travaille au début comme employé avec un salaire relativement faible et créé ensuite sa propre entreprise (Markakis, 1998 : 47). Il est célibataire : soit il cohabite avec des femmes autochtones avec lesquelles il a souvent des enfants, qu'il reconnaît néanmoins rarement, soit, une fois que sa situation se stabilise, il va chercher au pays une épouse (Markakis, 1998 : 50). En général il n'a qu'un faible niveau d'éducation ${ }^{18}$. Il élève ses enfants selon les traditions grecques, la communauté, l'école, les fêtes (religieuses et nationales) et l'église jouent un rôle important dans cette éducation.

«Leur première préoccupation était de combler leurs besoins religieux. Ils ont réussi en édifiant des églises orthodoxes (même dans les communautés grecques à faibles effectifs) et en faisant venir des prêtres de Grèce ou du Patriarcat œcuménique et du Patriarcat d'Alexandrie (pour le continent africain). La deuxième préoccupation fondamentale des immigrés était la mise en place, même dans un état rudimentaire, d'écoles grecques » (Hassiotis, 1993 : 180-181).

La deuxième génération grandit en apprenant le grec surtout à la maison. Les études grecques s'arrêtent à la fin du cycle de l'enseignement primaire à Yaoundé où se trouve l'école. Douala se trouve à 200 kilomètres et les déplacements sont particulièrement difficiles (même aujourd'hui). La plupart du temps ce sont les écoles françaises qui accueillent les élèves grecs généralement dès les petites classes. Pour justifier ce choix, les parents mettent en avant le fait que le système éducatif grec n'assure pas la continuité des études jusqu'à la fin de l'enseignement du second degré et qu'il est difficile pour un élève qui a suivi l'école primaire grecque de s'adapter ensuite à l'école française s'il souhaite poursuivre ses études dans l'enseignement secondaire. La deuxième génération parle le grec, mais ne l'écrit pas. Cependant, l'identité grecque de cette génération est très forte, et les mariages mixtes d'individus nés de parents grecs et ayant grandi au Cameroun sont très peu nombreux.

Les générations suivantes gardent des rapports importants avec la Grèce, mais les études se font en français et se poursuivent en général dans des universités françaises. Les mariages mixtes sont aussi plus nombreux (Métaxidès, 2004 : 327-341). Les jeunes parlent le grec dans leur environnement familial mais on observe que l'apprentissage de la langue est beaucoup plus facile chez les enfants dont les deux parents sont grecs et dans une moindre mesure chez ceux dont le père est grec et la mère est étrangère. Les contacts plus ou moins réguliers avec la Grèce rendent aussi plus aisé l'apprentissage de la langue.

17 Parmi eux les Koulaxides (au Cameroun depuis 1925), les Omérides (1929), les Tsekenis (1938), les Mavromatis (1928) et les Kalafatas (1930).

18 Si les immigrés originaires du Pont-Euxin et de l'Asie Mineure disposaient, pour la plupart, d'un bon niveau d'instruction ou d'une formation technique ainsi que d'une tradition familiale dans le commerce, ceux issus de la péninsule hellénique, partis pour des raisons économiques après la Deuxième Guerre mondiale, avaient en revanche un niveau d'éducation faible. 


\section{L'appartenance collective}

L'organisation communautaire des Hellènes en diaspora repose sur deux principales dimensions de l'appartenance grecque, la langue et la religion orthodoxe. L'éducation est donc, avec la pratique du culte, le premier souci de toute communauté grecque en diaspora (Prévélakis, 1996 : 53-68).

Durant les années d'après-guerre, les Grecs se sont structurés en deux communautés ${ }^{19}$, d'abord la Communauté de Douala puis celle de Yaoundé où ils ont construit leurs églises ${ }^{20}$. La ville de Yaoundé est la capitale et le centre administratif du pays, tandis que la ville de Douala est le grand port et le centre économique d'un secteur beaucoup plus large couvrant l'ensemble du golfe de Guinée. " La communauté grecque de Yaoundé et des environs » possède un bureau, une salle communautaire, une école primaire et une maison destinée à l'instituteur. Le siège de la Métropole orthodox $\mathrm{e}^{21}$ dispose d'un bureau et d'une résidence pour le Métropolite sur un terrain appartenant à la communauté. À Yaoundé existe également une mission orthodoxe grecque ainsi que le séminaire théologique «Hagios Markos » destiné à la formation des prêtres indigènes d'Afrique. L'église, en collaboration avec l'école grecque, organise, pour les Camerounais orthodoxes, des cours d'apprentissage du grec (Collectif, 2001 : 411-412). À Douala, la Communauté hellénique possède ses propres installations (salle communautaire, église, résidence du prêtre, installations sportives). On observe que l'acquisition des terres par les Grecs est liée à leur capacité d'adaptation à l'environnement local, au point qu'ils ne sont pas considérés comme des étrangers. Par exemple, Dimitras, tailleur de métier, originaire de l'île de Rhodes a épousé la fille du chef local Betoté AKWA. Cette relation entre un membre de la communauté grecque et la société locale a permis d'obtenir, de la part des chefs locaux, un terrain sur lequel se trouvent aujourd'hui les installations de la communauté grecque de Douala ${ }^{22}$.

19 « En vivant dans un environnement étranger et parfois hostile, les premiers immigrés grecs ont réalisé assez tôt que l'unité de la communauté était d'une importance capitale pour leur survie culturelle et sociale au sein de leur société d'accueil. Ainsi, la lutte contre l'assimilation était considérée à cette époque comme un élément indissociable de leur différenciation éducative, culturelle et nationale. Convaincus que seules les institutions et les organisations nationales officielles pouvaient les aider sur ces questions ils ont été amenés à fonder des communautés, des associations, des églises, qui, associées au sentiment national, à la religion orthodoxe et à l'éducation grecque, leurs ont permis de conserver la "grécité" vivante en Afrique du Sud durant tout le processus de l'évolution historique de leur communauté » (Mantzaris, 2004 : 227).

20 La Cathédrale de 1'Annonciation à Yaoundé (1955) ; Saints Constantin et Hellène à Douala (1959) ; les Chapelles de Taxiarhis et de Saint Yérassimos à Nkongsamba, construites l'une sur le terrain des installations Tzouvelos et l'autre sur les installations de Mavrommatis.

21 Un centre culturel fonctionne sous l'égide de la Sainte Métropole d'Accra. Ce centre culturel de l'Église orthodoxe organise des leçons de catéchisme, conférences, fêtes, messes et relations entre les communautés chrétiennes, ainsi que des actions sociales et philanthropiques. Le Conseil Central de la Métropole est une institution qui s'occupe des questions administratives et ecclésiastiques, sous la présidence du Métropolite. Les membres sont les représentants de la Communauté. Le Comité Central Économique et Technique s'occupe des affaires financières et de l'entretien des bâtiments de la mission ; il est composé du Métropolite, du Consul de Grèce, du président de la Communauté et des représentants de la communauté (Patriarcat d'Alexandrie, 1994 : 119).

22 L'information nous a été fournie par le vice-président de la Communauté Hellénique de Douala, Monsieur Alecos Nalbandidès, qui nous a offert un exemplaire du titre de propriété foncière (numéro 684) qui se trouve au bureau des hypothèques de la région du Wouri. 


\section{Éducation, tradition religieuse et identité nationale}

Selon Damanakis (1999 : 36), «la pédagogie traditionnelle appliquée en Grèce a adopté la définition politique du terme "identité nationale", elle l'a assimilé avec le terme de "conscience nationale" et l'a utilisé en tant qu' "idéal éducatif" pour l'éducation nationale » à l'intérieur et à l'extérieur des frontières grecques. Cette construction idéologique dominante peut cependant difficilement être associée à celle de la diaspora grecque.

Les Grecs au Cameroun ont conservé leur langue et leur grécité sans la présence de l'enseignement traditionnel grec. Les conjonctures n'ont pas permis aux communautés grecques du Cameroun de mettre en place un projet éducatif complet et l'État grec n'a pas cherché non plus à soutenir une telle perspective. L'école primaire de la communauté de Yaoundé n'était pas en mesure de garder ses élèves et les familles ont préféré offrir à leurs enfants une éducation qui permettait de poursuivre des études au collège et au lycée. Malgré ces inconvénients, les générations qui ont suivi les pionniers parlent grec (elles maîtrisent moins l'écrit) et celles qui ont suivi des études supérieures l'ont fait essentiellement en France. L'enseignement du français, de l'anglais et plus rarement d'un dialecte africain a permis aux jeunes de s'intégrer plus facilement à la diaspora cosmopolite grecque.

Le rôle de l'église, en tant qu'institution contribuant au maintien de la cohésion de la communauté grecque, dans toutes les régions de l'Afrique, est important ${ }^{23}$. Le patriarcat d'Alexandrie envoie des prêtres à chaque Métropole orthodoxe grecque d'Afrique ${ }^{24}$ et lorsque ce n'est pas possible, en raison du manque d'ecclésiastiques, c'est l'Église de Grèce qui apporte sa contribution. À partir du premier quart du XXe siècle ${ }^{25}$, la politique du Patriarcat, en dehors de son soutien aux populations grecques, vise également la diffusion de l'orthodoxie auprès des populations africaines et le développement des missions pour propager la foi. La principale conséquence de cette politique est l'augmentation du nombre de prêtres locaux qui prennent ainsi en charge les paroisses orthodoxes

23 « L'attachement des Grecs à l'orthodoxie était un facteur primordial pour conserver et renforcer la conscience et l'unité nationale. Les premiers immigrés grecs en Afrique du Sud ont compris très rapidement que l'édification de lieux de culte était une nécessité vitale » (Mantzaris, 2004 : 228).

24 «La circulation des prêtres, et en particulier des évêques, qui commencent leur carrière en Grèce et la poursuivent dans la diaspora, contribue au maintien du lien culturel et ethnique avec le territoire d'origine » (Bruneau, 2004 : 59).

25 Durant le XXe siècle, la renaissance intellectuelle du Patriarcat d'Alexandrie a atteint son aogée et le nombre des Grecs en Égypte a atteint son niveau le plus élevé. Le premier Patriarche du XXe siècle, Photios (1900-1925), a lutté pour la revalorisation du Patriarcat et a fondé sept nouvelles Métropoles en envoyant des ecclésiastiques très cultivés. C'est l'époque des grands bienfaiteurs de la Communauté grecque et du Patriarcat. Ils ont fondé de nouvelles institutions éducatives ainsi que des institutions culturelles. En 1908, le Patriarcat a inauguré ses propres installations d'imprimerie et il a lancé la publication de deux revues : Le phare de l'église et Pantainos. En 1926, Mélètios Metaxakis, élu Patriarche d'Alexandrie, a décidé de doter le Patriarcat de nouvelles structures. Il a désormais sous sa tutelle dix évêchés, quatre-vingt-dix églises, cinq monastères et 107 prêtres de paroisses. Le mandat du Patriarche, Christoforos (19391967) est une période de grands travaux et de modernisation. Plusieurs autres évêchés sont créés dans toute l'Afrique et la reconnaissance officielle des communautés orthodoxes africaines donne une forte impulsion aux activités des missionnaires (Collectif, 2001 : 377). 
africaines, atteignant même le rang hiérarchique de métropolites et d'évêques ${ }^{26}$. Bien que les Grecs ne soient plus dispersés dans les petites villes mais vivent principalement dans les grandes agglomérations urbaines, le manque de prêtres grecs, contribue à l'altération des liens entre les communautés grecques, déjà en déclin, et la tradition orthodoxe en tant que composante historique indissociable de l'hellénisme. Selon Michel Bruneau (2000 : 48), « La diaspora grecque africaine [...] liée à des structures héritées de la colonisation a un avenir incertain à moyen terme. Quant à son Église, elle sera probablement de plus en plus africaine et de moins en moins une institution de la diaspora ». L'existence de lieux de culte aujourd'hui en Afrique répond moins au besoin de se rassembler et de se retrouver entre compatriotes que d'affirmer de manière symbolique leur présence dans cet espace $^{27}$.

\section{Activités entrepreneuriales grecques en Afrique subsaharienne : différenciation culturelle ou adaptation?}

La capacité des Grecs à s'adapter dans des environnements difficiles, à vivre auprès des autochtones, à affronter les difficultés du climat et de la vie austère, n'est pas le trait principal qui leur a permis de se distinguer dans le domaine des affaires. Ce qui leur a permis de devenir des hommes d'affaires relevait d'une volonté de recherche d'opportunités qui pouvait les aider à s'affranchir de la dépendance des grandes sociétés d'exportation. Ils ont ainsi élaboré leur propre activité entrepreneuriale. Parallèlement, ils ont eu l'occasion, grâce au marché du cacao, de développer des petits commerces de détails en direction des populations locales.

Le développement du commerce d'exportation contrôlé par les colons européens a nécessité la création de lieux de stockage dans la ville et d'infrastructures urbaines. Les Grecs se sont chargés de créer les premières infrastructures de communication en achetant des voitures pour transporter les marchandises et les hommes. Le commerce et les transports sont ainsi devenus une activité particulièrement rentable investie par les hommes d'affaires grecs : commerce de détail, commerce en gros, commerce des denrées alimentaires, transports, cacao, café.

Le changement d'orientation des activités s'est fait durant la décennie 1970 (avec la crise pétrolière) puis au début des années 1980 (avec la libéralisation de l'économie et la mise en place de la politique d' « affaiblissement» de l'État au profit de l'initiative économique privée). Le développement des villes a vu naître de nouvelles activités et les Grecs vont investir de nouveaux secteurs d'activité mais aussi s'adapter à la concurrence des Libanais, des Indiens et des Camerounais (particulièrement les Bamiléké). Les Libanais continuent à travailler dans les transports, mais ils sont aussi très présents dans l'exploitation du bois et contrôlent de nombreux secteurs économiques dans les villes (boulangeries, quincailleries, grands magasins). Les Grecs ont abandonné le secteur des transports, leur

26 Son Éminence le Métropolite de Kambala et d'Ouganda Jonas et Monseigneur l'évêque de Bukoba Jéronimos sont des Africains.

27 «Les églises de la diaspora grecque reproduisent un modèle culturel historique de l'organisation de l'espace et du temps à travers son patrimoine. Elles n'essaient pas d'isoler les Grecs de la culture locale du pays d'accueil, ni de les éloigner du pays d'origine » (Prévélakis, 1996 : 220). 
présence dans le domaine de l'exploitation du bois est particulièrement limitée et, dans les villes, ils ont investi dans les mêmes commerces que les Libanais. Les deux communautés - libanaise et grecque - doivent aussi faire face à la concurrence des Indiens qui vendent des produits importés (principalement des produits manufacturés) provenant des pays asiatiques. Les Grecs accusent les Libanais de mener des affaires douteuses et les boulangers grecs tout particulièrement soupçonnent les Libanais de se lancer bien souvent dans des activités qui ne leur « appartiennent pas traditionnellement», dans le but de dissimuler leur participation au commerce parallèle de produits bon marché en utilisant leur pouvoir économique pour éviter les contrôles administratifs.

\section{LE TEMPS DU RETOUR AU PAYS D'ORIGINE}

Si l'on peut supposer que les raisons de la diminution du nombre de Grecs sont d'ordre économique, une analyse plus approfondie cependant montre que les motifs économiques ne peuvent pas à eux seuls expliquer ce phénomène. Nous constatons une baisse considérable des flux migratoires depuis la Grèce en même temps que l'augmentation du nombre de ceux qui retournent au pays, surtout à partir du milieu des années 1990. Ceux qui émigrent aujourd'hui au Cameroun peuvent être regroupés en deux catégories. La première comprend des personnes isolées qui décident d'emprunter la route difficile de l'émigration, soit pour des raisons personnelles, soit parce qu'elles ont vécu ou séjourné dans un pays africain voisin, pays qui n'est plus aujourd'hui accueillant et attractif pour eux. La seconde catégorie est constituée de personnes venues au Cameroun rejoindre des parents qui ont une activité économique dans le pays.

Les Grecs qui aujourd'hui sont actifs au Cameroun sont des enfants d'immigrés qui ne vivent que périodiquement dans le pays. Certains hommes d'affaires parmi eux font appel, à une main-d'œuvre originaire des pays de $1^{\prime}$ Est $^{28}$ qui souhaitent travailler en Afrique de façon provisoire. À part quelques exceptions, les Grecs qui ont été employés, quittent le pays au moment de leur retraite.

Le retour au pays est aussi lié à l'expiration des « droits » des Grecs du Cameroun sur la "propriété ». Ceux qui ont signé des contrats de longue durée (vingt/quarante ans) pour l'exploitation de terrains sur lesquels ils ont construit des entreprises se retrouvent dans l'obligation de les abandonner.

«Nous avons quitté définitivement le Cameroun en novembre 1997. J'ai laissé derrière moi ma fille et mon gendre, là-bas à mon travail. Ce travail est resté en place jusqu'au mois de mai 2000 [...]. Il y a un an maintenant que mon gendre est rentré, il a liquidé son travail et a mis en location nos bâtiments. C'est moi qui les ai construits avec un "bail", à savoir que le noir m'a donné le terrain, je l'ai exploité pendant vingt-cinq ans, mais pendant ces vingt-cinq années je lui versais un loyer pour le terrain, j'ai commencé à 700 000, il est monté à 880 000, il est

28 Ces personnes viennent d'Albanie, de Roumanie et des pays de l'ex Union Soviétique. Ils sont employés à des postes nécessitant un très fort rendement : caissiers de grandes surfaces, superviseurs de production dans les boulangeries, etc. Mais cela ne signifie pas que ces postes n'étaient pas pourvus en règle générale par des locaux. 


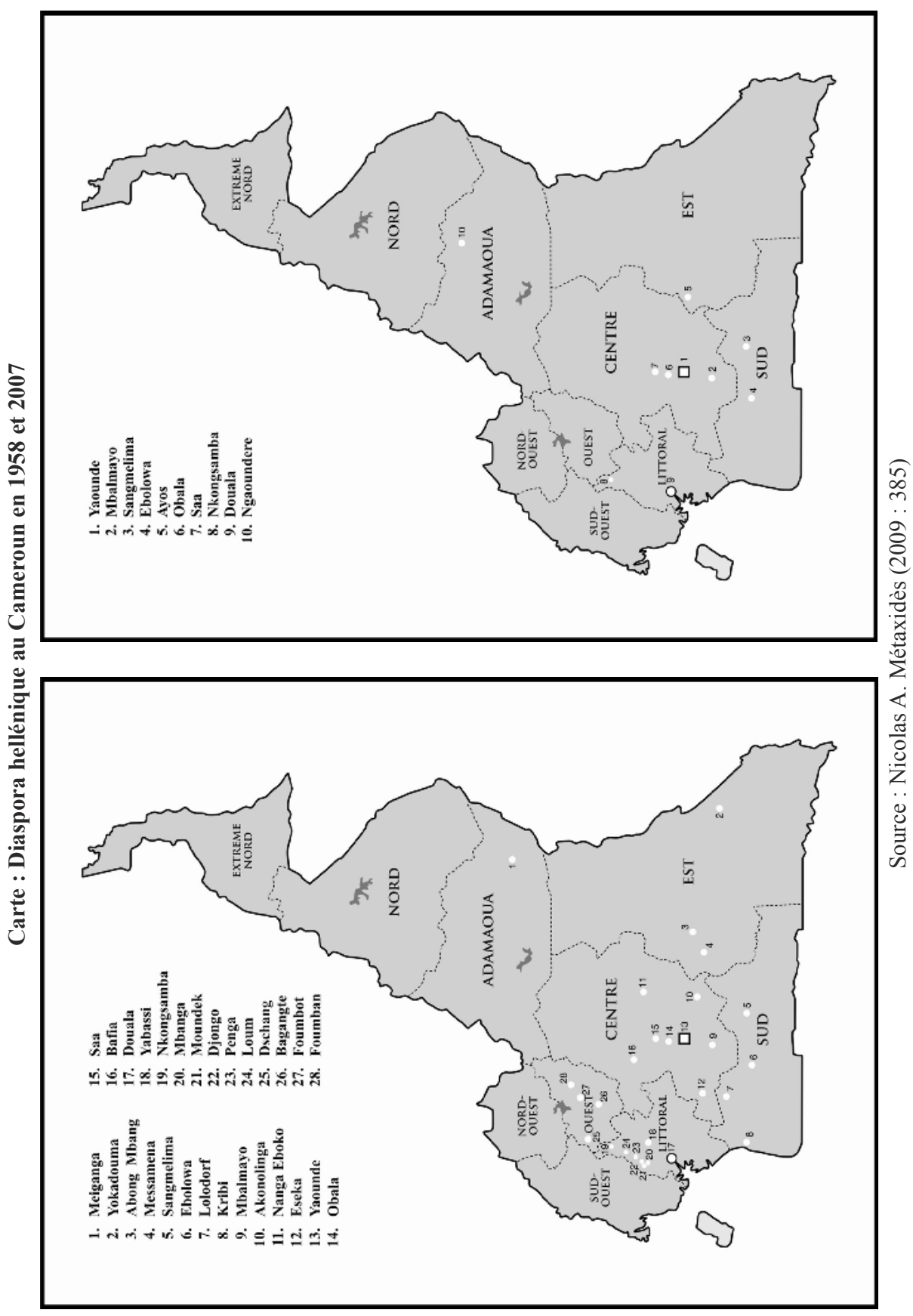


arrivé à 900000 et il montait conformément aux termes que nous avions inclus dans le contrat. Ce contrat est valable jusqu'en 2005. Après cette date, il est à lui. Mon gendre l'a loué à une société néerlandaise (jusqu'en 2005), après... Mais il y a aussi des Grecs qui ont acheté et qui ont construit. Puisque c'est à eux, ils sont obligés de rester. Maintenant ils essaient de les vendre pour une bouchée de pain, mais ils n'y arrivent pas. Les autres Européens n'en veulent pas. Le noir ne s'y intéresse pas, il se dit qu'un jour, ils les laisseront et ils partiront $»^{29}$.

Une autre raison qui conduit les plus jeunes générations de Grecs à ne pas vouloir vivre au Cameroun, mis à part un environnement professionnel difficile et même parfois hostile, est qu'elles sont dans l'incapacité de s'adapter à un milieu dont elles se sont éloignées avec leurs études en Europe ou aux États-Unis.

L'augmentation sensible du nombre de personnes qui retournent au pays à partir du milieu des années 1980 et le fait que, à partir de 1990, le nombre des Grecs qui quittent le Cameroun est supérieur au nombre de ceux qui cherchent à s'y établir, ont pour conséquence l'affaiblissement de la présence grecque.

\section{CONCLUSION}

Avant l'indépendance, les institutions étaient imposées par l'administration coloniale et protégeaient les activités des Européens reléguant les Africains dans des rôles subalternes et les utilisant principalement comme source de main-d'œuvre. Les Grecs se sont investis dans les secteurs de l'économie qui présentaient un intérêt pour les colons européens. Il s'agissait tout d'abord de la commercialisation de produits exportables (et dans certains cas des plantations de café, de bananes, etc.), puis, dans les transports de personnes et de marchandises contrôlant ainsi le circuit d'approvisionnement des grands centres urbains.

Les crises politiques (1957-1963 au Cameroun) ont eu des répercussions sur les activités des hommes d'affaires étrangers non africains (européens ou non) mais il est important de noter que cette période ne correspond pas à une réduction des effectifs de la communauté grecque. Selon les données officielles fournies par l'Annuaire Statistique de la Grèce, l'émigration des Grecs vers l'Afrique centrale s'est poursuivie.

Les Grecs, ainsi que les Libanais en Afrique occidentale et les Portugais au Zaïre, empêchaient les indigènes - en s'appuyant sur les réseaux d'affaires qu'ils avaient créés à l'époque coloniale - de pénétrer les secteurs économiques qu'ils contrôlaient. Par la suite les gouvernements africains ont soit nationalisé certaines sociétés, soit poussé à leur rachat, en partie ou en totalité, afin de favoriser l'émergence d'une élite locale d'entrepreneurs qui était en grande majorité une élite politique. Au début des années 1980, les États africains ont essayé de libéraliser les économies et de renforcer l'initiative privée. Les indigènes se sont chargés du commerce et des secteurs productifs abandonnés par les grandes sociétés européennes. Les communautés qui jouaient jusqu'alors le rôle d'intermédiaires (grecques et libanaises) sont passées du commerce des produits au commerce d'importation, à la

29 Entretien de Georges Chatzistamatis, accordé en février 2001. 


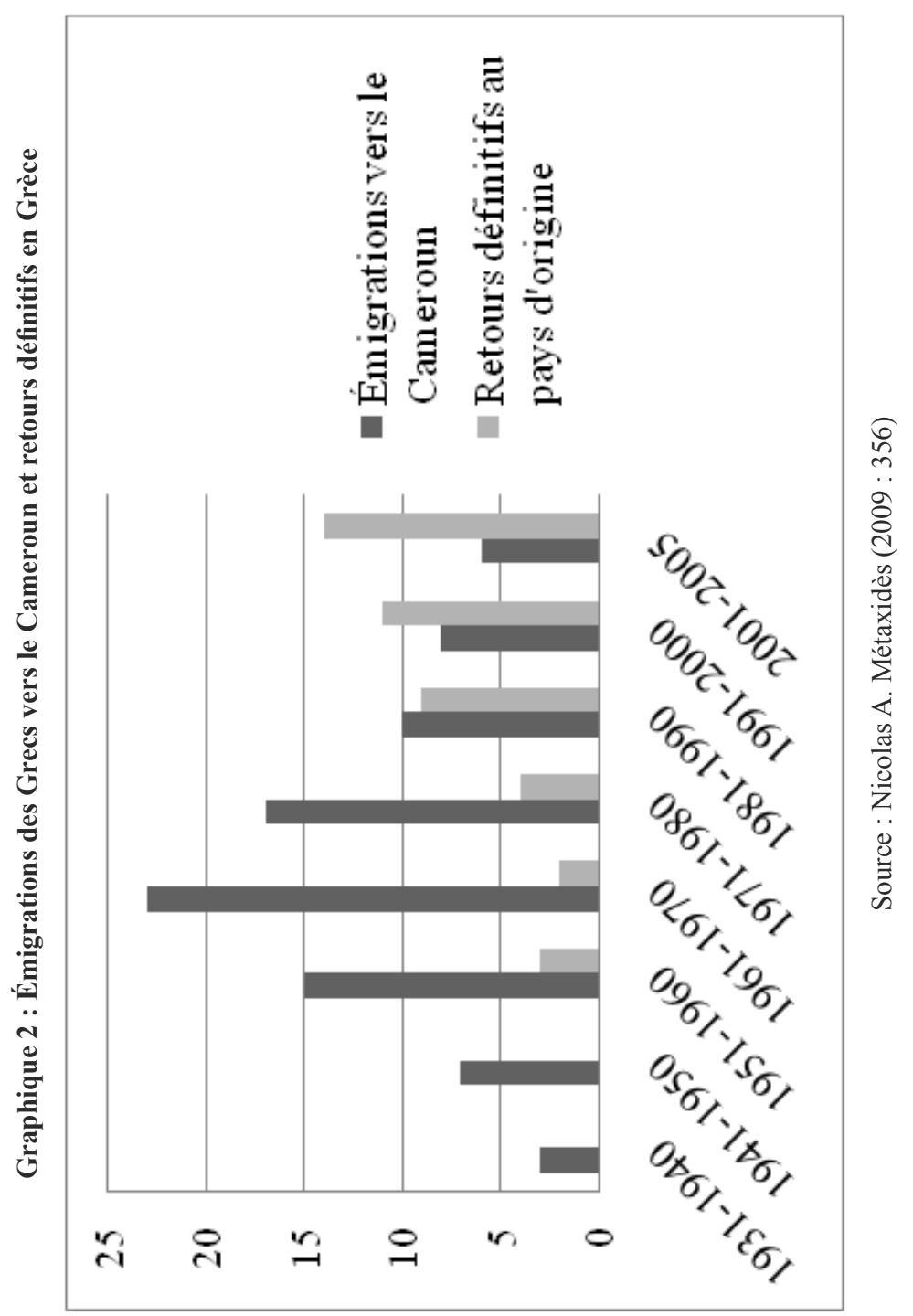


quincaillerie, au bâtiment, aux transports et aux boulangeries. Les marchés délaissés ont été récupérés par les commerçants locaux, mais aussi par les nouveaux arrivants en Afrique centrale et occidentale, les Indiens et les Chinois. Ils amènent avec eux leur savoir-faire commercial, acquis depuis l'époque où ils dominaient les échanges dans l'océan Indien et sur les côtes orientales de l'Afrique (Redding, 1991 ; Lachaier, 2003). Les Grecs, profitant de leurs relations avec le pouvoir politique local, avec le reste de la communauté française qui continue à occuper une place importante au niveau politique, ainsi que de la longue tradition économique qu'ils avaient dans la région, développent de nouvelles activités. D'abord dans des secteurs émergents comme le bâtiment, les constructions et la quincaillerie, puis comme les Libanais dans les boulangeries, le commerce des denrées alimentaires et enfin les grandes surfaces.

La diminution du nombre de Grecs en Afrique subsaharienne ne peut pas être attribuée uniquement aux conditions défavorables pour le développement des affaires et à l'incertitude sociale et politique. Elle est surtout due au fait que les enfants des Grecs délaissent les activités économiques familiales, alors qu'ils ont fait des études à l'étranger. Les Grecs de la seconde génération se revendiquent comme Grec, mais ne disposent pas de ressources culturelles suffisantes pour investir en Afrique noire. La survie du groupe et sa cohésion exigent dès lors une capacité d'adaptation et surtout une volonté de poursuivre le projet de ceux qui ont réussi dans le contexte local.

\section{Références bibliographiques}

AERTS Jean-Joël, COGNEAU Denis, HERRERA Javier, MONCHY Guy (de) et ROUBAUD François (2000) L'économie Camerounaise, un espoir évanoui, Paris, Karthala, 288 p.

AGATHAGELIDOU Marie (2003) La Paroikia grecque de Libye depuis les origines jusqu'aux années qui précèdent la Deuxième Guerre mondiale [en grec], Athènes, University Studio Press, $263 \mathrm{p}$.

BRUNEAU Michel (2000) Hellénisme et diaspora grecque. De la Méditerranée orientale à la dimension mondiale, Cahiers d'études sur la Méditerranée Orientale et le Monde TurcoIranien, 30, pp. 32-58.

BRUNEAU Michel (2001) Politiques de l'État-nation grec vis-à-vis de la diaspora, Revue Européenne des Migrations Internationales, 17 (3), pp. 9-22.

BRUNEAU Michel (2004) Diasporas et espaces transnationaux, Paris, Anthropos, 256 p.

COLLECTIF (2001) Atlas de la diaspora hellénique [en grec], tome II, Éditions Alexandre.

DAMANAKIS Michel (1999) Identité ethnoculturelle et enseignement dans la diaspora hellenique [en grec], in Éducation hellénophone à l'étranger, Actes du Congrès Panellénique et diasporique, Réthymnon, 26-28 Juin 1998, Laboratoire des Études Interculturelles et Migratoires (E. IIA.M.ME.), pp. 36-47.

HASSIOTIS Jean (1993) Revue de l'histoire de la diaspora hellénique [en grec], Éditions Vanias, $231 \mathrm{p}$.

KAZAKOS Panos et al. (1998) La diaspora grecque dans les pays d'Afrique [en grec], Athènes, Coédition du Ministère des Affaires étrangères de Grèce et du Secrétariat Général des Grecs de la Diaspora, 349 p.

KAZDAGHLI Habib (2000) Communautés méditerranéennes de Tunisie : les Grecs de Tunisie, du millet-i-rum à l'assimilation française : XVII-XXe siècles, Le Phare ecclésiastique, Revue théologique du Patriarcat d'Alexandrie, pp. 111-141. 
KAPTUE Léon (1987) Travail et main-d'œuvre au Cameroun sous régime français 1916-1952, Paris, L'Harmattan, $282 \mathrm{p}$.

LACHAIER Pierre (2003) Migrations indiennes en Afrique Orientale (Zanzibar, Tanzanie Tanganyika-, Kenya, Ouganda), Territoires et Circulations, pp. 1-13.

LANDA Janet (1993) Culture et activité entrepreneuriale dans les pays en développement : le réseau ethnique, organisation économique, in Brigitte Berger et al., Esprit d'entreprise, cultures et sociétés, Maxima, pp. 195-217.

LAZIDOU Hélène (2004) La diaspora grecque au Kenya et en Tanzanie : identité, formation et organisation en réseaux, Les Cahiers de l'Afrique de l'Est, 24, pp. 2-26.

LEMBEZAT Bertrand (1954) Le Cameroun, Paris, Éditions Maritimes et Coloniales, 157 p.

L'HELLÉNISME DE L'ÉTRANGER (édition mensuelle pour la communication des Hellènes de la diaspora), numéros 164 (1966), 209 (1970) et 280 (1976).

MACGAFFEY Janet (1987) Entrepreneurs and Parasites. The struggle for indigenous capitalism in Zaire, Cambridge University Press, 254 p.

MAINET Guy (1985) Douala, croissance et servitudes, Paris, L'Harmattan, 610 p.

MANTZARIS Evaggelos (1981) L'assimilation croissante d'une « nouvelle » petite bourgeoisie hellénique en Afrique du Sud [en grec], The Greek Review of Social Research, 42-43, pp. 145-152.

MANTZARIS Evaggelos (1984) The Greek miners in the Transvaal, South Africa: a socio-historical investigation, 1902-1913, Journal of the Hellenic Diaspora, XI (1), pp. 25-37.

MANTZARIS Evaggelos (1984) National and Social Consciousness in the Diaspora: the Greek Mine-Workers Union in South Africa 1905-1920, The Greek Review of Social Research, 48, pp. 94-110.

MANTZARIS Evaggelos (1987) Greek workers in South Africa: the case of the railway workers and cigarette-makers 1905-1914, Journal of the Hellenic Diaspora, XIV (3-4), pp. 49-63.

MANTZARIS Evaggelos (2004) Les Grecs en Afrique du Sud [en grec], in R. Clogg Dir., La Diaspora grecque au XXe siècle, Athènes, Ellinika Grammata, pp. 225-250.

MARKAKIS Jean (1998) Grecs en Afrique noire, 1890-1990 [en grec], Athènes, Trohalia, 154 p.

MARKANTONATOS L. (1965) La Nouvelle Afrique et la Grèce [en grec], Thessalonique, Zaharopoulos.

MÉTAXIDÈS Nicolas (2003) La présence hellénique en Afrique. Le cas des Grecs du Cameroun [en grec], in Histoire de la diaspora hellénique, Colloque International, Tome B', Réthymnon, 4-6 Juillet 2003, Laboratoire des Études Interculturelles et Migratoires (E.AIA.M.ME.), pp. 238-248.

MÉTAXIDĖS Nicolas (2004) Le phénomène de la diaspora hellénique en Afrique - son élaboration et ses perspectives (le cas des Grecs du Cameroun) [en grec], in Éducation interculturelle - le grec comme deuxième langue ou langue étrangère, 6e Colloque International, Tome II, Université de Patras, 20-22 juin 2003, KE.D.EK, pp. 327-341.

MÉTAXIDĖS Nicolas (2006) La diaspora hellénique en Afrique Centrale et Occidentale [en grec], in Les Grecs de la diaspora XV-XXIe siècles, Athènes, Parlement Grec, pp. 243-247.

MÉTAXIDĖS Nicolas (2009) Esprit d'entreprise et développement en Afrique subsaharienne, Thèse de doctorat, Université de Thessalie (Vólos, Grèce), 502 p.

MINISTĖRE DES AFFAIRES ÉTRANGÈRES DE GRÈCE (1980) La diaspora grecque [en grec], Athènes.

MINISTÈRE DES AFFAIRES ÉTRANGÈRES DE GRÈCE $(1987,1992)$ L'hellénisme à l'étranger [en grec], Athènes.

MONGO Beti (1971) Ville Cruelle, Présence Africaine (première édition1954), 223 p.

NATSOULAS Th. (1975) The Greeks in Ethiopia. Economic, political, and social life (1740-1936), $\mathrm{Ph}$. D., Syracuse University.

NGANDJEU Jean (1988) Le Cameroun et la crise, renaissance ou blocage? Paris, L'Harmattan, $167 \mathrm{p}$. 
PARIS Erato (2001) Les Grecs de Marseille dans la deuxième moitié du XIXe siècle. Une perspective nationale et transnationale, Revue Européenne des Migrations Internationales, 17 (3), pp. 23-42.

PATRIARCAT D'ALEXANDRIE (1994) Calendrier de l'Église d'Alexandrie, Alexandrie.

PATRIARCAT D'ALEXANDRIE (2007) Calendrier de l'Église d'Alexandrie, Alexandrie, année 97e.

PETRONOTI Marina (2000) Greeks in Osmara: Guardians of continuity, agents of change, Journal of the Hellenic Diaspora, 26 (1), pp. 7-20.

PRÉVÉLAKIS Marie (1996) L'Orthodoxie comme lien culturel, in G. Prévélakis Dir., Les réseaux des Diasporas, KYKEM, pp. 215-222.

PRÉVÉLAKIS Georges (1996) Les espaces de la diaspora hellénique et le territoire de l'Etat grec, in G. Prévélakis Dir., Les réseaux des Diasporas, KYKEM, L'Harmattan, pp. 53-68.

REDDING Gordon (1991) Culture et comportement entrepreneurial chez les Chinois d'outre-mer, in Brigitte Berger et al., Esprit d'entreprise, cultures et sociétés, Maxima, pp. 111-132.

SAKÉLLARIDÈS Georges (1960) Les Grecs de Rhodésie - Nyassaland, Afrique Centrale 1959-1960 [en grec], Athènes.

SOULOGIANNIS Efthimios (1999) La place des Grecs en Égypte [en grec], Athènes, Municipalité d'Athènes, $353 \mathrm{p}$.

SOULOGIANNIS Efthimios (2001) La communauté hellénique du Caire [en grec], Athènes, Kotinos, 260 p.

TREVASKIS Georges (1960) Eritrea. A Colony in Transition, Oxford Clarendon Press, http://info. grece.online.fr/.

VLAHOS Petros (1999) Si je savais... (autobiographie) [en grec], Athènes.1 